\title{
The Simplest Flowchart Stating the Mechanisms for Organic Xenobiotics-induced Toxicity: Can it Possibly be Accepted as a "Central Dogma" for Toxic Mechanisms?
}

\author{
Yeong-Chul Park', Sundong Lee ${ }^{2}$ and Myung-Haing $\mathrm{Cho}^{3}$ \\ ${ }^{1}$ GLP Center, Center, Catholic University of Daegu, Keongsan, Gyeongsangbuk-do, Korea \\ ${ }^{2}$ Dept. of Preventive Korean Medicine, School of Korean Medicine, Sangji University, Wonju, Korea \\ ${ }^{3}$ Laboratory of Toxicology, College of Veterinary Medicine, Seoul National University, Seoul, Korea
}

(Received July 11, 2014; Revised August 29, 2014; Accepted September 18, 2014)

\begin{abstract}
Xenobiotics causing a variety of toxicity in biological systems could be classified as two types, inorganic and organic chemicals. It is estimated that the organic xenobiotics are responsible for approximately $80 \sim 90 \%$ of chemical-induced toxicity in human population. In the class for toxicology, we have encountered some difficulties in explaining the mechanisms of toxicity caused especially by organic chemicals. Here, a simple flowchart was introduced for explaining the mechanism of toxicity caused by organic xenobiotics, as the central dogma of molecular biology. This flowchart, referred to as a central dogma, was described based on a view of various aspects as follows: direct-acting chemicals vs. indirect-acting chemicals, cytochrome P450-dependent vs. cytochrome P450-independent biotransformation, reactive intermediates, reactivation, toxicokinetics vs. toxicodynamics, and reversibility vs. irreversibility. Thus, the primary objective of this flowchart is to help better understanding of the organic xenobiotics-induced toxic mechanisms, providing a major pathway for toxicity occurring in biological systems.
\end{abstract}

Key words: Biotransformation, Cytochrome P450, Reactive intermediates, Toxicokinetics vs. toxicodynamics, Reactivation, Reversibility vs. irreversibility

\section{INTRODUCTION}

Toxicology is simply defined as the study of the adverse effects of xenobiotics on living organisms. A xenobiotic is a general term referring to any chemical foreign to an organism or, in other words, any compound not occurring within the normal metabolic pathways of a biological system $(1,2)$. Even if xenobiotics include organic or inorganic chemicals, it has been supposed that organic xenobiotics are greatly responsible for chemical-induced toxicity in human population. This may be due to the organic xenobiotics that are the basic constitutive chemical for most medications and poisons for living things (3). In addition, more than $90 \%$ of Group I agents, which are carcinogenic to humans, are

Correspondence to: Yeong-Chul Park, GLP Center, Catholic University of Daegu, 13-13 Hayang-ro, Hayang-eup, Gyeongsan-si, Gyeongsangbuk-do 712-702, Korea

E-mail: ycpark@cu.ac.kr

This is an Open-Access article distributed under the terms of the Creative Commons Attribution Non-Commercial License (http:// creativecommons.org/licenses/by-nc/3.0) which permits unrestricted non-commercial use, distribution, and reproduction in any medium, provided the original work is properly cited. derived from organic xenobiotics (4).

Toxic mechanisms, of organic xenobiotics, have been explained on the basis of a cascade of events starting with exposure, proceeding through distribution and metabolism, and ending with the interaction with cellular macromolecules. This process is simply expressed as toxicokinetics and toxicodynamics (1). However, understanding the mechanisms underlying toxicity of xenobiotics in living system requires considering the molecular and biochemical reactions involved, as well as the relationships of ultimate toxic metabolites with cellular macromolecules (5). Thus, teaching and understanding for toxic mechanisms can sometimes be a difficult task, partly because of their inherent difficulty and partly due to the complex interrelation among many factors such as a type of ultimate toxicant and cellular molecules in the presence or absence of biotransformation.

For better teaching and easier understanding about organic xenobiotics-induced mechanism of toxicity, some flowcharts with hypothetical descriptions have been developed and used (6-9). However, these descriptive flowcharts are useful only for explaining partly or limited processes in the whole toxic mechanisms, because of no reflection with toxicokinetics and toxicodynamic perspectives. In addition, most 
of them were focused only on the toxic mechanisms by indirect-acting xenobiotics requesting biotransformation. Thus, the flowchart considering toxicokinetic and toxicodynamic perspectives in the presence of, or the absence of biotransformation is necessary to cover the whole process of organic xenobiotics-induced toxic mechanisms.

Here, the simplest flowchart was introduced to facilitate teaching and better understanding about organic xenobiotics-induced mechanisms of toxicity. For a simple but core descriptive flowchart, the key steps and characteristics in xenobiotics-induced toxic process were considered as follows: direct-acting chemicals vs. indirect-acting chemicals, biotransformation, cytochrome P450-dependent vs. cytochrome $\mathrm{P} 450$-independent biotransformation, reactivation, reactive intermediates, and reversibility vs. irreversibility. This descriptive flowchart, is called "Central Dogma of Toxic Mechanism for Organic Xenobiotics", which is similar to the concept from central dogma of molecular biology, and was developed by modifying the original flowchart in Korean (10). Thus, the primary objective of this flowchart is again to introduce the central dogma explaining a major pathway for organic xenobiotics-induced toxicity in English.

\section{CENTRAL DOGMA}

The simlest flowchart, a cenntral dogma, was already introduced from the previous work contained in the Korean textbook The Molecular and Biochemical Principles of Toxicology (10). This simplest flowchart was explained again by other additional works for organic xenobiotics-induced toxicity.

Central dogma of toxic mechanism for organic xenobiotics. The simplest flowchart is shown in Fig. 1 from which all of the toxic mechanisms for organic chemicals can be explained. The simplest flowchart or central dogma of toxic mechanisms for organic xenobiotics was supported by the following aspects: direct-acting chemicals vs. indirect-acting chemicals, biotransformation, cytochrome P450dependent vs. cytochrome P450-independent biotransformation, reactive intermediates, reactivation, toxicokinetics vs. toxicodynamics and reversibility vs. irreversibility.

Direct-acting chemicals vs. indirect-acting chemicals. Organic xenobiotics which induce the adverse effect or toxicity in the biological system can act either directly or indirectly $(11,12)$. In general, organic xenobiotics entering the biological system are subject to biotransformation. Here, biotransformation refers to changes in xenobiotic compounds as a result of enzymatic reactions. Organic xenobiotics are biotransformed in several organs, but mainly in the liver. Since most organic xenobiotics undergo biotransformation prior to their toxic action, this process is identified in the flowchart as a major pathway. However, a few organic xenobiotics are not subject to biotransformation prior to their action; this process is identified in the flowchart as a minor pathway. Thus, organic xenobiotics are classified by either direct-acting or indirect-acting chemicals according to the presence or absence of biotransformation. Usually direct-acting xenobiotics undergo natural decomposition by hydrolysis, resulting in the chemical modification identified as an active form, and then display electrophilic property. This electrophilic property has a key role in interacting with cellular macromolecules such as protein, lipid and DNA, causing toxicity inside organisms. If the parent compound has an electrophilic property, the reaction of the parent compound with the environmental material would happen, prior to entering inside the organism. Of course, without any chemical modification, some parent compounds which act directly on the target molecules. These xenobiotics show their similar action to some endogenous compounds such as a hormone, inducing and causing an alteration in a physiological

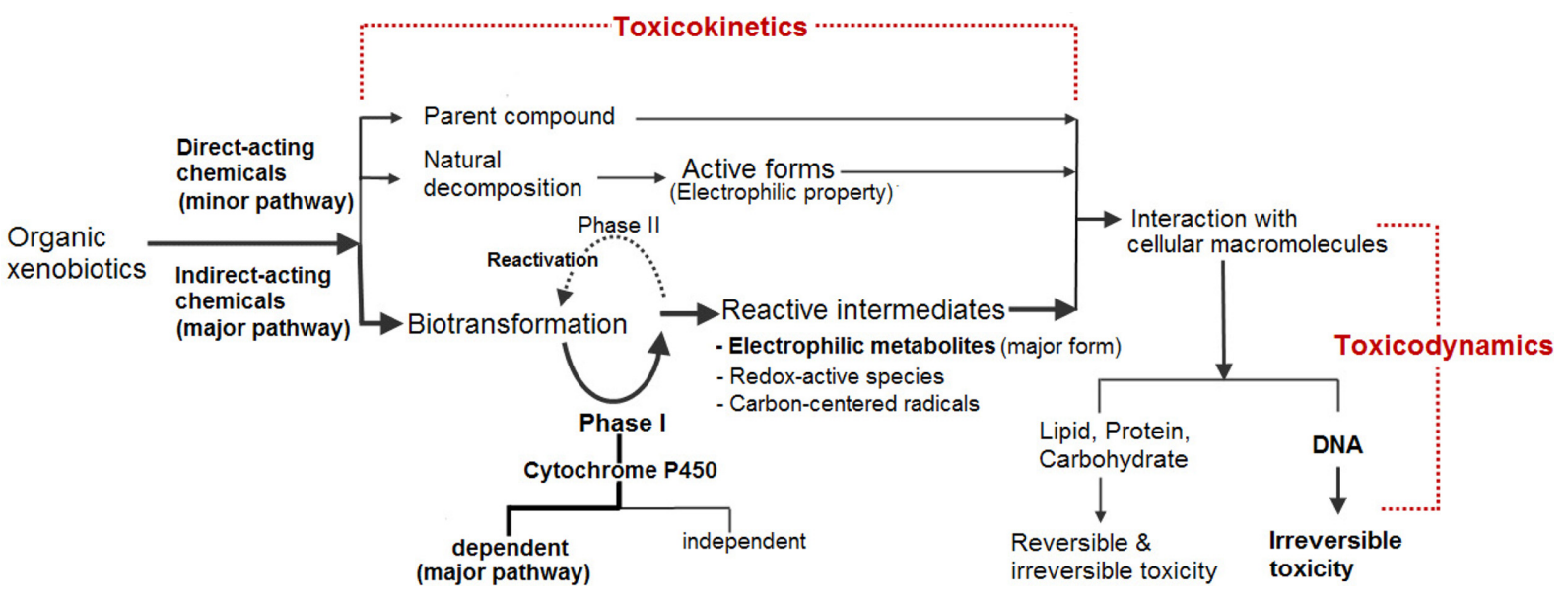

Fig. 1. The simplest flowchart stating the mechanism for organic xenobiotics-induced toxicity*.

*: Adapted from Park, 2010. 
control mechanism. Direct-acting chemicals are those which can "immediately" react with a substance because the parent chemical is chemically reactive. Conversely, indirect-acting chemicals are not initially reactive and so they require an activation step such as biotransformation. In general, it is believed that a great majority of toxic effects are caused by indirect-acting chemicals, including most medicines and environmental pollutants.

Biotransformation. Biotransformation, sometimes referred to as metabolism, is the structural modification of a chemical by enzymes in the body. Organic xenobiotics are biotransformed in several organs including the liver being the most important one. Organic xenobiotics absorbed in the gastrointestinal tract must pass through the liver, where they can be biotransformed and thus eliminated before being distributed to other parts of the body. Thus, biotransformation of a xenobiotic can dominate toxicokinetics, and the metabolites may reach higher concentrations in organisms than their parent compounds (13). In addition, toxicodynamics, dealing with the mechanisms by which toxicants produce unique cellular effects within the organism, are related to biotransformation producing toxic metabolites like reactive intermediates (1). Thus, it can be surmised that the biotransformation process can dominate toxicokinetics and toxicodynamics of most xenobiotics. The enzymes in the biotransformation process play a major role in the chemical conversion of xenobiotics. There are two main groups of biotransformation enzymes according to a series of two reactions: Phase I reaction and phase II reaction. Phase I reactions normally add a functional group to the xenobiotic which enables the Phase II reaction to take place $(14,15)$. Phase I reactions are carried out by oxidative enzymes like the cytochrome P450 and the flavin monooxygenases, as well as hydrolytic enzymes like esterases and amidases $(6,16,17)$. The group for the phase II reaction is composed of enzymes that catalyze the conjugation of these functionalized compounds to a water-soluble moiety (e.g. glucuronic acid, sulfate, glutathione) (6).

Cytochrome P450-dependent vs. cytochrome P450independent biotransformation. In Phase I reaction, almost $80 \%$ of organic xenobiotics are biotransformed by cytochrome P450 enzymes, referred to as cytochrome P450-dependent biotransformation (17-19). In fact, from the 315 most commonly prescribed drugs in the US, 175 $(56 \%)$ are primarily cleared by cytochrome P450-dependent biotransformation (20). In humans, to date 57 different P450 isoforms have been identified, which were assigned to 18 families and 43 subfamilies based on their protein sequences (18). From these 18 families, the CYP3A subfamily, which comprises CYP3A4, 3A5 and 3A7, is responsible for more than $50 \%$ of cytochrome P450-mediated biotransformation (21). Thus, cytochrome P450-dependent biotransformation is classified as a major pathway for xenobiotics biotransformation, especially in the liver.

Reactive intermediates. In general, biotransformation results in detoxification of xenobiotics and is accompanied by the formation of chemically stable metabolites. However, in some cases, these reactions can convert xenobiotics to toxic metabolites that are chemically reactive (22). This process is commonly referred to as metabolic activation or bioactivation. Thus, bioactivation is the biotransformation process by which a xenobiotic may be converted to more reactive or toxic forms. These reactive or toxic forms are referred to as reactive intermediates, such as electrophilic metabolites having electron-seeking property, redox-active species having the ability to accept and donate electrons during redox-cycling reaction, and carbon-centered radicals having unpaired valence electrons at carbon (8,23-25). These reactive intermediates may be capable of either modifying biological material covalently or participating in redox-cycling reactions leading to the toxic effects. The continuous presence of chemically reactive intermediates can lead to adverse effects and various disease conditions (26).

In addition, most of reactive intermediates are formed in a direct or indirect relation with cytochrome P450 activity (8,19,27-29). Thus, cytochrome P450-dependent biotransformation is a major factor in determining toxicity. This is especially true regarding electrophilic metabolites, as they are the most important reactive intermediates. Many of the chemical-induced toxicities have been known to be associated with their ability to react with a variety of targets within the cell $(26,30)$. The toxic effects by electrophilic metabolites are initiated by the covalent binding to cellular macromolecules, such as proteins, and nucleotides within DNA, forming various adducts $(24,31,32)$. However, electrophilic metabolites and other reactive intermediates formed during bioactivation process may be also detoxified efficiently by glutathione conjugation and other Phase II reactions $(32,33)$. Thus, toxic effects occur only when the balance between the production of reactive metabolites and their detoxification is disrupted (34). Although a substantial body of information is available on the biotransformation of xenobiotics to reactive metabolites and chemical nature of reactive intermediates, less is known about how reactive intermediates interact with cellular macromolecules or constituents and how those interactions cause cell injury and death (35). Thus, it is believed that a great majority of toxic effects are caused by reactive intermediates generated by cytochrome P450-dependent biotransformation of xenobiotics $(8,19)$.

Reactivation. After Phase II reaction, a conjugated xenobiotic or a conjugated reactive intermediate is generally associated with detoxification. An unusual occurrence 
was noted: when the reactive intermediate was conjugated, especially with glutathione after Phase I, it was activated again under specific circumstances with reactions such as hydrolytic reaction of glutathione, and cytochrome P450dependent biotransformation again in other tissues and organs $(10,36)$. This process is referred to as reactivation, which induces toxic effects. In addition, most reactive intermediates are formed by Phase I metabolic pathways, however, in very rare cases Phase II pathways can also generate reactive metabolites (8). For example, there are known xenobiotics that, upon conjugation with sulfate or glucuronic acid, are converted to chemically reactive metabolites leading to toxicity (37).

Toxicokinetics vs. toxicodynamics. In general, four potential processes exist for a chemical interacting with an animal: absorption, distribution, biotransformation and excretion, referred to as toxicokinetics. However, the biotransformation of a xenobiotic may be the most important process in toxicokinetics, since kinetic profiles of a xenobiotic in the body can change its structural modification at this point. In addition, the reactive intermediate's chemical reactivity and vicinity are important factors affecting the toxicokinetics, because the reactive intermediate may be able to interact with the biological target in the site where biotransformation occurs. If toxicokinetics shows the quantitative relationship among absorption, distribution, biotransformation, and excretion of xenobiotics and their metabolites, toxicodynamics is defined as the characterization of the molecular, biochemical and physiological effects of xenobiotics and their reactive intermediates. Toxicodynamics describes the dynamic interactions of xenobiotics and their reactive intermediates with cellular macromolecules known as the site of action. Cellular macromolecules such as proteins, ion channels, DNA, or a variety of other receptors are a biological target of xenobiotics and their reactive intermediates $(24,38)$. The mechanism of action of xenobiotics and their reactive intermediates, as determined by their chemical properties, will determine what cellular macromolecules or receptors are targeted and the overall toxic effect at the cellular level and organismal level. Thus, toxicodynamics can be explained in combination with toxicokinetics in determining the potential effects and toxic mechanisms.

Reversibility vs. irreversibility. In a view of toxicodynamics of reactive intermediates, the two types of interaction with cellular macromolecules are reversible and irreversible. Reversible interactions are not permanent and can be changed, otherwise, returned to normal. Most toxic effects are reversible and do not cause permanent damage, but complete recovery may take a long time. However, irreversible interactions are permanent and cannot be changed once they have occurred. Some reactive intermediates cause irreversible interaction with cellular macromolecules, espe- cially DNA. Many procarcinogens biotransformed by cytochrome P450 enzymes to electrophilic metabolites can react irreversibly with DNA, causing mutations and eventually, carcinogenesis (22,37). In addition, interaction of reactive intermediates with the nervous system is usually irreversible since its cells cannot divide and be replaced. Accordingly, relatively small changes in overall xenobiotic biotransformation, particularly in increasing the formation or decreasing the detoxification of the reactive intermediate can have a major effect on tissue exposure and toxicity (30). It is known that the amount of reactive intermediate formed during the biotransformation of most xenobiotics generally constitutes only a small fraction, perhaps $1 \% \sim 10 \%$ of the total xenobiotic disposition (30). However, the toxicological potential of such a small fraction of reactive intermediates is further compounded by the irreversible nature of interaction with other DNA, which results in multistagecarcinogenesis by the accumulation over time of DNA molecular lesions $(10,39)$.

\section{CONCLUSION}

The central dogma of molecular biology, represented by three major stages, replication, transcription and translation, has long been used in helping to explain the flow of genetic information within a biological system. In addition, it has been a beneficial contribution towards understanding the basic concept of molecular biology in much easier terms even when exceptions are discovered; for example, reverse transcriptase, an enzyme that uses RNA as a template to form DNA (40-42). In a case of toxicology, even though tremendous progress has been made in past decades, elucidating the mechanisms for organic xenobiotics-induced toxicity, there are still no descriptive flowcharts sufficient enough to cover the whole process of organic xenobioticsinduced toxic mechanisms in a view of toxicokinetics and toxicodynamics. As shown in Fig. 1. the simplest flowchart was introduced to facilitate teaching and better understanding organic xenobiotics-induced mechanisms of toxicity. All mechanisms of organic xenobiotics-induced toxicity can be explained with this flowchart, so this is why we refer to it as the Central Dogma. However, it is believed that a great majority of toxic effects are caused by reactive intermediates, especially electophilic metabolites, generated by cytochrome P450 through biotransformation of a parent xenobiotic. This process in producing reactive intermediates is classified as a major pathway in a view of toxicokinetics. As a major pathway in toxicodynamics, there will be interactions of reactive intermediates with cellular macromolecules, resulting in permanently irreversible changes. Finally, it is our expectation that this flowchart will be replaced by other promoted as the true Central Dogma for organic xenobiotics-induced toxicity so that all people can easily approach the field of toxicology. 


\section{ACKNOWLEDGMENTS}

This project was supported by the Korea institute of toxicology (KIT). The author wishes to thank Professor Leonard G. Matarese of the Catholic University of Daegu for his generous time in reviewing this manuscript.

\section{REFERENCES}

1. Evans, T.J. (2013) Small Animal Toxicology (Third Edition), Chapter 2 - Toxicokinetics and Toxicodynamics, Elsevier Publishing Company, pp. 13-19.

2. Nagarkatti, P.S. and Nagarkatti, M. (1987) Immunotoxicology: Modulation of the immune system by xenobiotics. Def. Sci. J., 37, 235-244.

3. Borzelleca, J.F. (2000) Profiles in toxicology. Paracelsus: herald of modern toxicology. Toxicol. Sci., 53, 2-4.

4. International Agency for Research on cancer (IARC) Monograph. (2014) Overall Evaluations of Carcinogenicity: An Updating of IARC Monographs. 1, 109

5. Betharia, S., Corcoran, G.B. and Ray, S.D. (2014) Mechanisms of Toxicity: In Encyclopedia of Toxicology (3rd Ed), Elsevier Publishing Company, pp. 165-175.

6. Liska, D.J., Lyon, M. and Jones, D.S. (2006) Detoxification and biotransformation imbalance, Explore, 2, pp. 112-140.

7. Gregus, Z. and Klaassen, C.D. (2008) Casarett \& Doull's Toxicology (7th ed), The Basic Science of Poisons, Unit 1: Basic principle of toxicology, Chapter 3: mechanisms of toxicity, MC GRAW Hill, pp. 35-81.

8. Attia, S.M. (2010) Deleterious effects of reactive metabolites. Oxid. Med. Cell. Longevity, 3, 238-253.

9. Wells, P.G., Bhullera, Y., Chen, C.S., Jeng, W., Kasapinovic, S., Kennedy, J.C., Kim, P.M., Laposa, R.R., McCallum, G.P., Nicol, C.J., Parman, T., Wiley, M.J. and Wong, A.W. (2005) Molecular and biochemical mechanisms in teratogenesis involving reactive oxygen species. Toxicol. Appl. Pharmacol., 207, 354-366.

10. Park, Y.C. (2010) The molecular and biochemical principles of toxicology. Korean studies Information Publishing Company, Korea, pp. 19-24.

11. Kumar, V., Abba, A.K. and Aster, J.C. (2012) Robbins Basic Pathology (7th Edition), Elsevier, pp. 199-200.

12. Working, P.K. (1989) Mechanistic Approaches in the Study of Testicular Toxicity: Agents that Directly Affect the Testis. Toxicol. Pathol., 17, 452-456.

13. Ashauer, R., Hintermeister, A., O'Connor, I., Elumelu, M., Hollender, J. and Escher, B.I. (2012) Significance of xenobiotic metabolism for bioaccumulation kinetics of organic chemicals in gammarus pulex. Environ. Sci. Technol., 46, 3498-3508.

14. Liska, D.J. (1998) The Detoxification Enzyme Systems. Altern. Med. Rev., 3. 187-198.

15. Kumar, G.N. and Surapaneni, S. (2001) Role of drug metabolism in drug discovery and development. Med. Res. Rev., 21, 397-411.

16. Schroer, K., Kittelmann, M. and Lütz, S. (2010) Recombinant human cytochrome P450 monooxygenases for drug metabolite synthesis. Biotechnol. Bioeng., 106, 699-706.
17. Guengerich, F.P. (2006) Cytochrome P450s and other enzymes in drug metabolism and toxicity. AAPS J., 8, E101E111.

18. Zanger, U.M. and Schwab, M. (2013) Cytochrome P450 enzymes in drug metabolism: Regulation of gene expression, enzyme activities, and impact of genetic variation. Pharmacol. Ther, 138, 103-141.

19. Orhan, H. and Vermeulen, N.P. (2011) Conventional and novel approaches in generating and characterization of reactive intermediates from drugs/drug candidates. Curr. Drug Metab., 12, 383-394.

20. Bertz, R.J. and Granneman, G.R. (1997) Use of in vitro and in vivo data to estimate the likelihood of metabolic pharmacokinetic interactions. Clin. Pharmacokinet., 32, 210-258.

21. Yan, Z. and Caldwell, G.W. (2001) Metabolic profiling, and cytochrome P450 inhibition \&induction in drug discovery. Curr. Top. Med. Chem., 1, 403-425.

22. Srivastava, A., Maggs, J.L., Antoine, D.J., Williams, D.P., Smith, D.A. and Park, B.K. (2010) Role of reactive metabolites in drug-induced hepatotoxicity. Handb. Exp. Pharmacol., 196, 165-194.

23. Bolton, J.L., Trush, M.A., Penning, T.M., Dryhurst, G. and Monks, T.J. (2000) Role of quinones in toxicology. Chem. Res. Toxicol., 13, 135-160.

24. Lopachin, R.M. and Decaprio, A.P. (2005) Protein Adduct Formation as a Molecular Mechanism in Neurotoxicity. Toxicol. Sci., 86, 214-225.

25. Nioi, P. and Hayes, J.D. (2004) Contribution of NAD(P)H: quinone oxidoreductase 1 to protection against carcinogenesis, and regulation of its gene by the Nrf2 basic-region leucine zipper and the arylhydrocarbon receptor basic helix-loophelix transcription factors. Mutat. Res., 555, 149-171.

26. Guengerich, F.P. (1992) Metabolic activation of carcinogens. Pharmacol. Ther., 54, 17-61.

27. Goetz, M.E. and Luch, A. (2008) Reactive species: A cell damaging rout assisting to chemical carcinogens. Cancer Lett., 266, 73-83.

28. Gram, T.G. (1997) Chemically reactive intermediates and pulmonary xenobiotic toxicity. Pharmacol. Rev., 49, 297-341.

29. Tolando, R., Zanovello, A., Ferrara, R., Iley, J.N. and Manno, M. (2001) Inactivation of rat liver cytochrome P450 (P450) by $\mathrm{N}, \mathrm{N}$-dimethylformamide and $\mathrm{N}, \mathrm{N}$-dimethylacetamide. Toxicol. Lett., 124, 101-111.

30. Well, P.G., Kim, P.M., Nicol, C.J., Parman, T. and Winn, LM. (1997) Drug Toxicity in Embryonic Development I; Reactive Intermediates. Springer Berlin Heidelberg Publishing Company, 124, 453-518.

31. Gold, B., Marky, L.M., Stone, M.P. and Williams, L.D. (2006) A review of the role of the sequence-dependent electrostatic landscape in DNA Aakylation patterns. Chem. Res. Toxicol., 19, 1402-1414.

32. Shu, Y.Z., Johnson, B.M. and Yang, T.J. (2008) Role of biotransformation studies in minimizing metabolism-related liabilities in drug discovery. AAPS J., 10, 178-192.

33. van Bladeren, P.J. (2000) Glutathione conjugation as a bioactivation reaction. Chem. Biol. Interact., 129, 61-76.

34. Masubuchi, N., Makino, C. and Murayama, N. (2007) Prediction of in vivo potential for metabolic activation of drugs into chemically reactive intermediate: correlation of in vitro and in 
vivo generation of reactive intermediates and in vitro glutathione conjugate formation in rats and humans. Chem. Res. Toxicol., 20, 455-464.

35. Djurovic, J. (2012) Biotransformation of the toxic chemical substances. Math. Models Methods Appl. Sci., 91-95.

36. Smith, M.T., Yager, J.W., Steinmetz, K.L. and Eastmondt, D.A. (1989) Peroxidase-dependent metabolism of benzene's phenolic metabolites and its potential role in benzene toxicity and carcinogenicity. Environ. Health Perspect., 82, 23-29.

37. Glatt, H. (2000) Sulfotransferases in the bioactivation of xenobiotics. Chem. Biol. Interact., 129, 141-170.

38. Guengerich, F.P. and Shimada T. (1998) Activation of procar- cinogens by human cytochrome P450 enzymes. Mutat. Res., 400, 201-213.

39. Ashauer, R. and Escher, B.I. (2010) Advantages of toxicokinetic and toxicodynamic modeling in aquatic ecotoxicology and risk assessment. J. Environ. Monit., 12, 2056-2061.

40. Crick, F. (1970) Central dogma of molecular biology. Nature, 227, 561-563.

41. Thieffry, D. and Sarkar, S. (1998) Forty years under the central dogma. Trends Biochem. Sci., 23, 312-316.

42. Mattick, J.S. (2004) The hidden genetic program of complex organisms. Sci. Am., 291, 60-67. 